\title{
Nuevas perspectivas de la educación física: tendencias, contenidos, métodos y evaluación en los municipios de Neiva y Pitalito
}

\author{
Jazmín Andrea Olaya Muñoz \\ Universidad Surcolombiana, Colombia \\ Jazandolay@hotmail.com \\ John Freddy Molina Rodríguez \\ Universidad Surcolombiana, Colombia \\ furialaboyana2012@hotmail.com
}

\section{Resumen}

La investigación denominada "Tendencias pedagógicas vigentes de la clase de educación física del nivel de básica secundaria y media en las instituciones educativas públicas de los municipios de Neiva y Pitalito" constituyo en una investigación que pretendió analizar, descubrir, narrar y caracterizar los ambientes de aprendizaje actuales que se asocian y contribuyen al desarrollo y fortalecimiento de la Educación Básica en los municipios de Neiva y Pitalito; además, la necesidad de conocer las experiencias educativas por parte de los maestros en su práctica, conocer el tipo de tendencia utilizada, es decir, si el docente tiene en cuenta aspectos técnicos, de fundamentación, observa habilidades y destrezas por parte del estudiante y el desempeño que éste tenga.

También, se consideró fundamental establecer en la actualidad cómo los docentes desarrollan sus currículos o contenidos, métodos, formas de trabajo y evaluación, que predominan en la clase, cómo se está desarrollando el proceso educativo y cuáles son las matices (modelos pedagógicos, estrategias didácticas, enfoques metodológicos) que implementan los docentes en sus clases, frente a innumerables alternativas que se pueden desarrollar en el área de Educación Física. El proyecto se realizó partiendo de de la imperiosa necesidad que se da desde el ámbito internacional, nacional, departamental y municipal de llevar a cabo estudios que visualicen la realidad educativa que se presenta en este contexto.

Palabras Clave: Tendencias Pedagógicas, Educación Física, Formas de Trabajo, Programación Curricular y Evaluación.

\section{New perspectives of physical education: trends, content, methods and evaluation in the municipalities of Neiva and Pitalito}

\footnotetext{
Abstract

The research called "Current pedagogical trends of the physical education class of secondary and secondary level in public educational institutions of the municipalities of Neiva and Pitalito" constituted a research that sought to analyze, discover, narrate and characterize learning environments current that are associated and contribute to the development and strengthening of Basic Education in the municipalities of Neiva and Pitalito. Further, they need to know the educational experiences on the part of the teachers in their practice, to know the type of tendency used, that is, if the teacher takes into account technical aspects, of foundations, observes skills and abilities on the part of the student and the performance that it has.

Also, it was considered essential to establish at present how teachers develop their curricula or contents, methods, forms of work and evaluation, which predominate in the class, how the educational process is being developed and what the nuances are (pedagogical models, teaching strategies, methodological approaches) that teachers implement in their classes, in front of innumerable alternatives that can be developed in the area of Physical Education. The project was carried out based on the urgent need that exists from the international, national, departmental and municipal levels to carry out studies that visualize the educational reality presented in this context.
} 
Key words: Pedagogical Trends, Physical Education, Work Forms, Curricular Programming and Evaluation.

\section{Introducción}

En las últimas dos décadas, países de todo el mundo han demostrado importantes avances en materia de educación a través de la implementación y adecuación de distintos programas que procuran encontrar alternativas para que los docentes cuenten con una variedad de mecanismos que permitan establecer estrategias de mayor calidad, pertinencia, cobertura y eficiencia en el área de Educación Física.

"En el ámbito internacional, autores como Contreras Jordán Onofre, Pieron M. Rozengardt Rodolfo y Sánchez Bañuelos entre otros, plantean la necesidad de superar los estilos de clase tradicionales basados en el maestro y en consecuencia abogan por el desarrollo de una Educación Física con enfoque constructivista y por la implementación de estilos de clase que fomenten la creatividad e impliquen cognitivamente al estudiante" (Camacho Coy \& Amaya Bahamon, 2011)

En el contexto del desarrollo del conocimiento la Educación Física construye sus propias transformaciones en concepciones y prácticas. De esta forma los procesos de investigación y de experiencia docente han permitido identificar las tendencias de la Educación Física que se presentan no de manera pura y aislada, sino con interacciones en la práctica educativa, para contribuir fielmente al proceso educativo de esta área del conocimiento tan vital para el ser humano.

Siguiendo estos planteamientos, en el año 2004 México asume para la Educación Física el Enfoque "Motriz de integración dinámica", que propicia en los docentes, "un cambio de actitud metodológica, que se hace evidente cuando el alumno se convierte en el centro del proceso educativo, sin limitar la participación del maestro" (Secretaría de Educación Pública, 2004)

Lo que evidenció la importancia de hacer la revisión de las tendencias pedagógicas de la Educación Física, conocer acerca de cómo los docentes la utilizan en el área y así formular y diseñar estrategias que permitan innovar los currículos que se orientan en cada uno de los grados; además, permitirá que el docente plantee estrategias de interaccion dinamica para construir clases innovadoras, que promuevan en el alumnado una mayor participación en la clase.

En el ámbito nacional, los lineamientos curriculares estructurados por el Ministerio de Educación nacional plantean que la Educación Física debe tener un enfoque pedagógico que retome principios de la pedagogía crítica y el constructivismo pedagógico, y en tal sentido precisa:

"El enfoque curricular plantea un giro hacia una Educación Física que exprese lo multidimensional y lo heterogéneo, en función de una acción educativa que corresponda a las exigencias de un nuevo mundo y un nuevo sujeto. Que replantee sus conceptos, prácticas y metodologías y los ubique en una perspectiva que atienda los procesos de formación personal y social y potencie hacia nuevas significaciones la dimensión corporal y lúdica del ser humano" (Ministerio de Educación Nacional, 2010)

En el Departamento del Huila la Universidad Surcolombiana junto con la Secretaría de Educación Departamental, han sido pioneras en el diseño e implementación de programas curriculares para la Educación Física en los niveles de preescolar, básica primaria, secundaria y media. Esto ha permitido que a nivel regional se cuente con propuestas en cada uno de los niveles educativos citados, los cuales se han estructurado con la participación de los docentes y han contado con fases de difusión y acompañamiento.

En el año 2010, un estudio realizado por el Grupo de Investigación Acción Motriz de la Universidad Surcolombiana, dio a conocer a través de resultado de investigación denominada “Caracterización Pedagógica de la Clase de Educación Física, realizada en el departamento del Huila", donde los resultados mostraron ciertas contradicciones entre lo que manifiestan los docentes en las entrevistas, lo que se registra en las programaciones y lo evidenciado en los diarios de campo. 
Según el estudio, la tendencia que más predomina para los maestros del Departamento del Huila es: "la tendencia integradora o de complementación propuesta en la Alternativa Curricular para la Educación Física, propia de una pedagogía crítica y constructivista". (Camacho Coy $\mathcal{E}$ Amaya Bahamon, Una mirada al Área de Educación Física en el Departamento del Huila, 2011). Cabe destacar que ésta investigación se desarrolló con los docentes participantes en instituciones del sector público del departamento del Huila, se tomó una institución del Municipio de Neiva.

Para la investigación, se definió como muestra a los docentes de 10 instituciones del sector público del Municipio de Neiva y 5 instituciones del sector público del Municipio de Pitalito, con ellos se indagó acerca de las siguientes variables del área de Educación Física: contenidos que orientan, la metodologia que implementa el docente y la evaluación que éste desarrolla en clase.

El grupo investigador analizó que, aunque los docentes manifestaron el uso frecuente de la tendencia integradora o de complementación, en el proceso de observación fueron diferentes los resultados, debido a que, en el desarrollo de las clases y los registros en los diarios de campo, es evidente la vigencia de métodos tradicionales propios de la tendencia deportiva.

"Además la investigación también mostró cómo en las instituciones educativas del departamento del Huila a nivel general, no se tienen definidos los modelos pedagógicos en los PEI, algunas instituciones hacen referencia a modelos participativos, tradicionales y de modificabilidad cognitiva, entre otros, pero no existe un consenso sobre el particular. Razón por la cual, la tendencia pedagógica definida para el área se hace de manera aislada frente a los fundamentos pedagógicos de la institución, cada área del conocimiento, define sus parámetros y de esta manera se pueden llegar a presentar contradicciones en los procesos de enseñanza, evaluación, seguimiento de estudiantes, entre otros". (Camacho Coy E Otros, 2011).

Resultado que evidencia que en la mayoría de los casos, los docentes siguen orientando las clases con las tendencias que más predoniman, se continua con el tradicionalismo, metodologías de trabajo fuera de contexto, contenidos y procesos de evaluación similares a las de décadas atrás, no se presentan avances, innovación, procesos de formación diferentes, ni currículos novedosos en el área.

Teniendo en cuenta lo anterior, se convierte en un objetivo imprescindible, el indagar y establecer los ambientes de aprendizaje que permitan determinar y estructurar las tendencias pedagógicas que se orientan en el área de Educación Física, y así relacionar contenidos, enfoques, tendencias, metodologías y procesos de evaluación en el área de Educación Física, lo que le permitirá a los estudiantes recibir clases motivadoras, con un alto grado de goce, y se interesen por ser partícipes activos de las actividades que desarrollará el docente. Cabe destacar que la investigación mencionada anteriormente fue realizada en el departamento del Huila, donde Neiva y Pitalito no constituyó muestra principal, sino por el contrario, se tuvo en cuenta cada uno de los otros municipios pertenecientes al departamento. Es por ello no que existe ningún estudio a nivel de estos municipios.

Gracias a investigaciones desarrolladas a nivel regional, nacional e internacional se han establecido las tendencias pedagógicas de la Educación Física, con el fin de mejorar el proceso de enseñanza y aprendizaje, y conocer cuáles son las más utilizadas actualmenteen las instituciones educativas de los municipios de Neiva y Pitalito. Surge entonces la necesidad de dar respuesta a la siguiente pregunta de investigación: "cuáles son las tendencias pedagógicas de la clase de educación física que se encuentran vigentes en los niveles de básica secundaria y media que se desarrollan en las instituciones educativas públicas de los municipios de Neiva y Pitalito"

\section{Aspectos Metodológicos}

\section{Tipo de Estudio}

El desarrollo del presente estudio se enmarcó dentro del ámbito descriptivo transversal, dado que se indago sobre de las Tendencias Pedagógicas, la Programación Curricular, los Métodos y Formas de Trabajo, y los Procesos de Evaluación del Estudiante; se establecieron comparaciones entre los conceptos dados por los 
docentes, en las entrevistas y en el análisis de las programaciones.

Desde esta perspectiva la investigación "Tendencias pedagógicas vigentes de la clase de Educación Física, del nivel de básica secundaria y media, en las instituciones educativas públicas de los municipios de Neiva y Pitalito" metodológicamente hablando, hizo énfasis en:

Involucro de manera activa en el estudio a docentes, estudiantes y directivos docentes, de las instituciones educativas seleccionadas para el estudio.

Centro como "objeto de conocimiento y finalidad" de la investigación, las prácticas desarrollados en las clases en el nivel de básica secundaria y media para analizar e interpretar sus dinámicas de funcionamiento.

Vinculo a los docentes, como Coinvestigadores (sujeto investigador) y a partir de sus vivencias y experiencia en el contexto de la práctica educativa (objeto de investigación) encontrar alternativas que contribuyan a mejorar la Educación Física de los municipios de Neiva y Pitalito.

\section{Población y Muestra}

La población estuvo constituida por las instituciones educativas oficiales de los Municipios de Neiva y Pitalito del Departamento del Huila, certificadas con estudiantes de grados $6^{\circ}$ a $11^{\circ}$ y además se incorporan los docentes encargados de orientar el área de Educación Física; distribuida de la siguiente manera.

Tabla 1. Instituciones Educativas del Sector Público del Municipio de Neiva Seleccionadas en la Muestra

\begin{tabular}{|l|l|}
\hline No. & INSTITUCIÓN EDUCATIVA \\
\hline 1. & I.E. INEM JULIAM MOTTA SALAS \\
\hline 2. & I.E. LUIS IGNACIO ANDRADE \\
\hline 3. & I.E. NACIONAL SANTA LIBRADA \\
\hline 4. & I.E. INSTITUTO TECNICO SUPERIOR \\
\hline 5. & I.E. JOSE EUSTACIO RIVERA \\
\hline 6. & I.E. OLIVERIO LARA BORRERO \\
\hline 7. & I.E. NORMAL SUPERIOR DE NEIVA \\
\hline 8. & I.E. JUAN DE CABRERA \\
\hline 9. & I.E. GABRIEL GARCIA MARQUEZ \\
\hline 10. & I.E. HUMBERTO TAFUR CHARRY \\
\hline
\end{tabular}

Tabla 2. Instituciones Educativas del Sector Público Municipio de Pitalito Seleccionadas en la Muestra

\begin{tabular}{|l|l|}
\hline No. & INSTITUCIÓN EDUCATIVA \\
\hline 1. & I.E.M. NACIONAL \\
\hline 2. & I.E.M MONTESSORI \\
\hline 3. & I.E.M.HUMBERTO MUNOOZ ORDOÑEZ \\
\hline 4. & I.E.M. WINNIPEG \\
\hline 5. & I.E.M. LICEO SUR ANDINO \\
\hline
\end{tabular}

La muestra estuvo compuesta por diez instituciones educativas oficiales del Municipio de Neiva y cinco instituciones educativas oficiales del Municipio de Pitalito del Departamento del Huila, donde participó directamente un docente de Educación Física encargado de orientar el área.

Las instituciones fueron seleccionadas de manera criterial. Hicieron parte de la muestra 10 docentes de Educación Física del Municipio de Neiva y 05 docentes de Educación Física del Municipio de Pitalito. El margen de error estimado fue del $0.3 \%$ y el nivel de confiabilidad del 95\%; calculado mediante el programa estadístico MacStat 2.0.

"Las instituciones fueron seleccionadas según muestreo no probabilístico, dado que: "la elección de los elementos no depende de la probabilidad, sino de causas relacionadas con las características de la investigación o de quien hace la muestra. Aqui el procedimiento no es mecánico ni con base en fórmulas de probabilidad, sino que depende del proceso de toma de decisiones de un investigador o de un grupo de investigadores $y$, desde luego, las muestras seleccionadas obedecen a otros criterios de investigación". (Hernández Sampieri, Fernández Collado, E Baptista Lucio, 2010)

Para el caso de los estudiantes en la aplicación de encuestas y teniendo en cuenta que en el Municipio de Neiva son más los educandos, fueron aplicadas 1.080 encuestas y 450 encuestas para el Municipio de Pitalito. Los estudiantes seleccionados pertenecen a las Instituciones seleccionados en la muestra inicial, y contaron con los siguientes criterios de inclusión:

Estar matriculados en una Institución Educativa pública

Estar cursando un grado entre $6^{\circ}$ y $11^{\circ}$ 
Por parte de las Instituciones Educativas se seleccionaron teniendo en cuenta los siguientes criterios:

Que pertenecieran a los Municipios de Neiva y Pitalito, ubicados en las diferentes zonas urbanas del mismo.

Que los docentes que orientan el área de Educación Física en dichas instituciones fueran licenciados en Educación Física.

Que las instituciones participantes hicieran parte del sector oficial

\section{Etapas del Estudio}

\section{Fase de Socialización}

En esta etapa se realizó un primer acercamiento con los docentes y directivos docentes de las instituciones educativas que pertenecían a la muestra. Permitió dar a conocer a las personas participantes, los aspectos conceptuales del proyecto, los objetivos, las variables de estudio y las acciones a seguir en el desarrollo del mismo.

Fase de Trabajo de Campo

El trabajo de campo comprendió de manera general las siguientes acciones:

Realización de visitas a cada una de las instituciones seleccionadas. Estas se realizaron en el primer semestre académico del año 2016A para desarrollar el proceso socialización y posteriormente se llevaron a cabo tres visitas a cada una de las instituciones, para su respectiva aplicación de los instrumentos y sistematización delos mismos. Las actividades que se cumplieron en cada institución fueron las siguientes:

Desarrollo de entrevistas las cuales se aplicaron a cada docente seleccionado. La entrevista fue grabada en medio magnético y posteriormente se realizó la transcripción a medio físico. La entrevista se desarrolló en un espacio y hora previamente concertada con el profesor.

Lectura de documentos sobre programaciones curriculares, para lo cual se solicitó al profesor los documentos pertinentes. Esta lectura se llevó a cabo en un sitio diferente a la institución educativa visitada, lo cual implico la autorización para fotocopiar documentos y retirarlos. A partir de la lectura se llenó una ficha de registro confrontada con los profesores para precisar datos y aclarar dudas.

Aplicación de Encuestas a los estudiantes, quienes fueron escogidos con un muestreo criterial.

\section{Proceso de Recolección de la Información}

La información fue recolectada de manera fundamental durante las visitas, y se llevaron a cabo tres visitas por institución educativa.

Las entrevistas fueron semiestructuradas y grabadas en medio magnético, se llevaron a cabo en un lugar cómodo dentro de la institución educativa. Su duración está calculada en una hora aproximadamente y se pueden realizar varias sesiones si el desarrollo de la temática lo amerita.

Fueron desarrolladas por el grupo de estudiantes de la Maestría en Educación Física, quienes poseen experiencia en la realización de la investigación y desarrollarán actividades previas de preparación en la realización de entrevistas.

Las fichas de registro se utilizaron para sistematizar los aspectos sobre planificación y programación curricular de la Educación Física, y se diligenciarán a medida que se va realizando la lectura de los documentos suministrados por los docentes.

\section{Resultados}

La investigación fue desarrollada durante el año 2016-A y 2016-B y se mostraran los resultados más significativos, con relación a las entrevistas realizadas, las programaciones curriculares analizadas y las encuestas aplicadas a los estudiantes, actores pertenecientes a las instituciones públicas de Neiva y Pitalito. En su interpretación se entrelazaron las respuestas y así conocer lo expuesto por los docentes y estudiantes participantes en la investigación.

Para el buen desarrollo de la investigación $y$ exposición de los resultados fue necesario 
exponer cada una de las variables establecidas, las cuales desde el inicio del proyecto fueron definidas así: Tendencias Pedagógicas, Programación Curricular, Métodos y Formas de Trabajo, y Evaluación.

\section{Tendencias Pedagógicas}

Con respecto a la variable relacionada con Tendencias Pedagógicas, se parte de la necesidad de dar respuesta a la pregunta de investigación y para ello mediante un mapa conceptual se mostrarán los aportes dados por los docentes, así como también cuál es el modelo pedagógico que implementa la Institución Educativa, permitir criterios generales para el área que sirvan de orientación a los enfoques de cada una de los contenidos que conforman la programación curricular en la Institución. Por lo anterior es importante destacar que mostraremos los resultados de la Encuesta de acuerdo a lo que el estudiante nos dijo en sus respuestas.

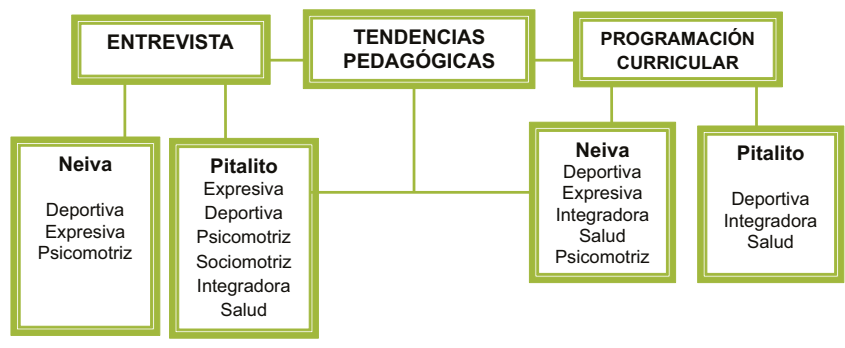

Mapa Conceptual 1. Tiene una Tendencia Definida para el Área

Con respecto a las entrevistas se obtuvo que para el municipio de Neiva, sólo tres docentes manifestaron el uso de una tendencia en sus clases de Educación Física y los restantes no tienen claridad al respecto y desconocen las Tendencias Pedagógicas del área. Para el municipio de Pitalito, tanto en las entrevistas como en las programaciones curriculares los docentes manifiestan hacer uso de las tendencias pedagógicas, por esto es notorio que existe coherencia en la programación curricular, como en la entrevista realizada, de igual manera para el Municipio de Neiva, en las Programaciones Curriculares se exponen cuatro tendencias que se observaron durante el análisis del instrumento.

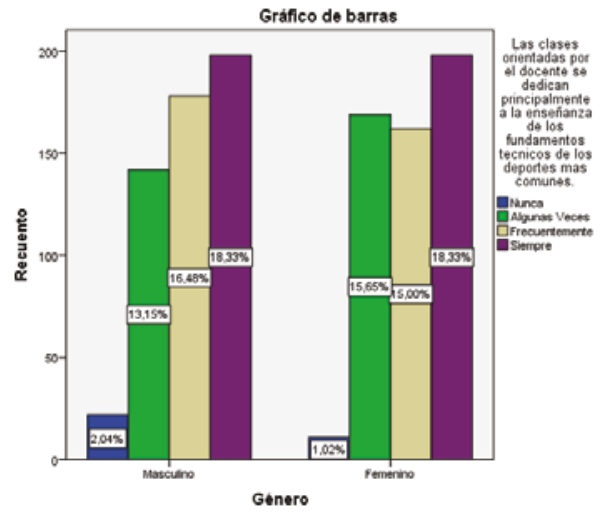

Ilustración 1. Tendencia más utilizada para el Municipio de Neiva

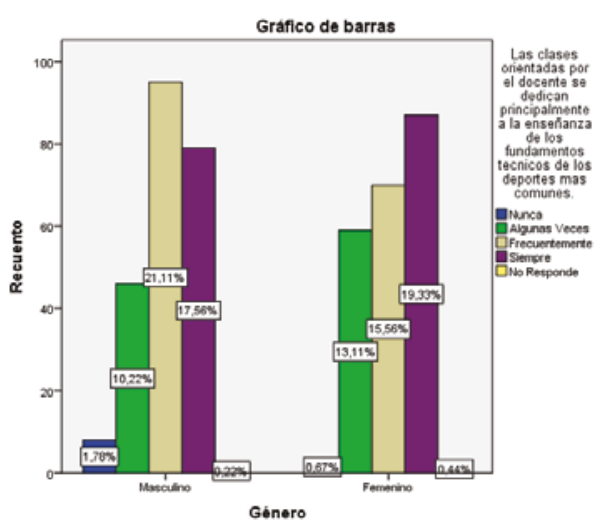

Ilustración 2. Tendencia más utilizada para el Municipio de Pitalito

El consolidado establece que en su mayoría, tanto mujeres como hombres afirman que la tendencia utilizada por el docente Siempre se dirige principalmente al desarrollo de actividades dedicadas a la enseñanza de los fundamentos técnicos de los deportes más comunes, con un $36,66 \%$ para Neiva y un $36,89 \%$ para Pitalito en ambos géneros. De igual manera, un buen porcentaje asume que Frecuentemente el docente trabaja los deportes (con el 31,48\% en Neiva y $36,67 \%$ para Pitalito entre mujeres y hombres en ); quedando así un porcentarje del menos del 5\% para Nunca.

Es por ello que en los tres instrumentos se considera que la tendencia más utilizada es la Deportiva. Lo manifiestan los estudiantes, los docentes y el análisis de las programaciones curriculares; sinembargo, existedesconocimiento por parte de los docentes al no manifestar qué tendencia utilizan y al mencionar otro tipo 
de concepto que no hace relación alguna con las tendencias consideradas para el presente estudio.

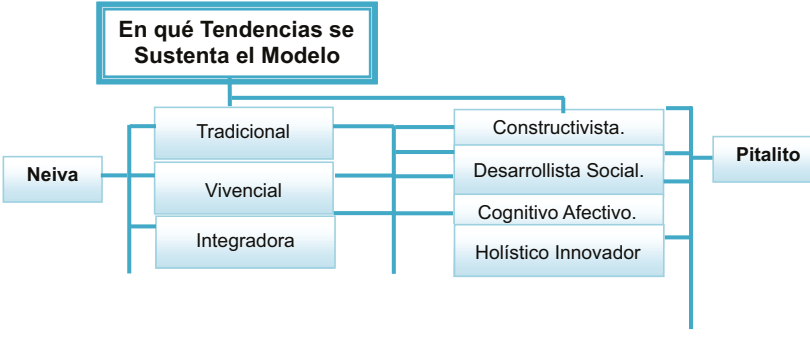

Mapa Conceptual 2. Cómo considera su aporte en la Adopción de esta Tendencia

Los docentes en la Entrevista manifestaron que la Tendencia que ellos utilizan se basa en: Tradicional, Vivencial o Integradora para el Municipio de Neiva, para el Municipio de Pitalito, conocen el modelo pedagógico que posee la institución educativa, dando como resultado una estrecha relación en las entrevistas y sus programaciones curriculares.

Es por ellola necesidad de formar profesionales íntegros y con conocimientos claros y precisos en cuanto a la fundamentación teórica y bases epistemológicas desde el nacimiento de la Educación Física; es notorio el vacío de la historia, de conceptos, y todo lo relacionado con el área.

\section{Programación Curricular}

En el área de Educación Física y en las demás áreas de conocimiento se hace necesario la implementación de una programación curricular, lo que le permite al docente planear y programar sus contenidos y/o temáticas que deben ser desarrolladas en el transcurso del semestre académico o año lectivo, y es allí donde todo docente de Educación Física prima por planear sus actividades, con fines de llegar y orientar sus conocimientos y no improvisar en una clase donde es el estudiante nuestro actor fundamental y debe éste trabajar activamente con gozo y armonía.

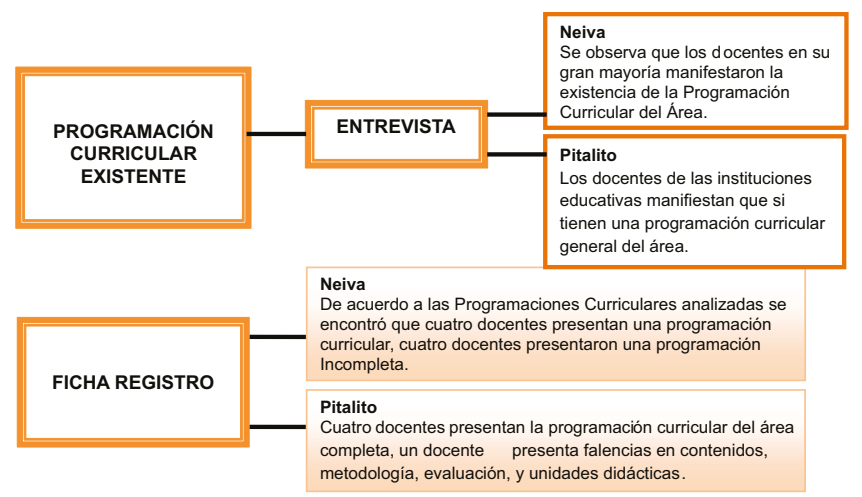

Mapa Conceptual 3. Existe una Programación Curricular General del Área

Cabe resaltar que, para el Municipio de Neiva, no se hizo el análisis a dos programaciones curriculares dado que su estructura no fue clara y se consideró que el docente en realidad no cuenta con una programación general para el área. Para el Municipio de Pitalito se observó con claridad que, de acuerdo a los lineamientos de la Secretaria de Educación Municipal, las instituciones educativas cuentan con su respectiva programación desde los grados sextos a once.

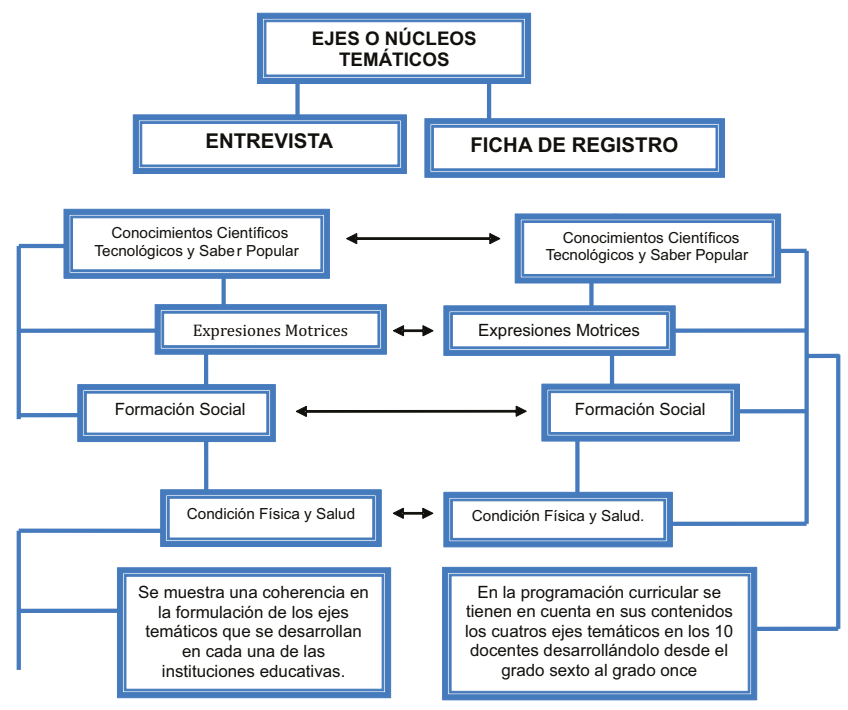

Mapa Conceptual 4. Cuáles son los ejes temáticos que se tienen en cuenta en la programación general del área

Tanto para los Municipios de Neiva y Pitalito, se observó que existe coherencia por parte de los docentes tanto en las programaciones curriculares como en la entrevista, dado que trabajan los mismos ejes temáticos para el área; ejes temáticos planteados en la Alternativa Curricular del área de Educación Física, por 
docentes de Educación Física de la Universidad Surcolombiana. Sin embargo se resalta que para el Municipio de Pitalito, tres de los cinco docentes presentaron unos ejes temáticos similares, sino que son descritos de manera diferente. Se concluye que existe directa relación entre lo que se desarrolla en el campo deportivo y lo planteado en las programaciones curriculares.

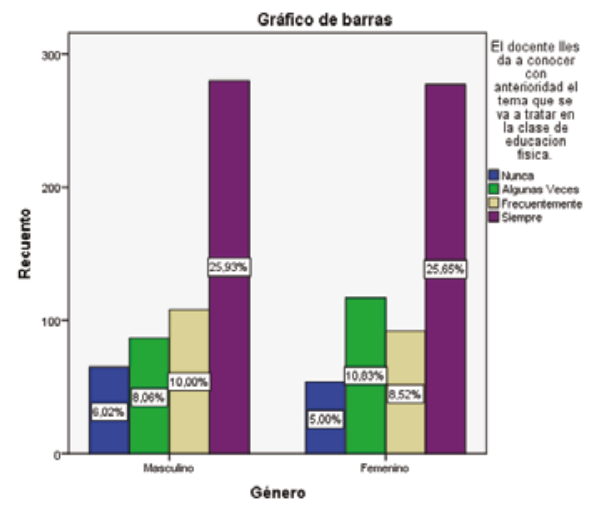

Ilustración 3. El docente les da a conocer con anterioridad el tema que se va a tratar en la clase de Educación Física

El consolidado establece que en su mayoría afirma que el docente sí les da a conocer con anterioridad el tema que se va a tratar en la clase de Educación Física, con un 51,58\% en mujeres y hombres que afirman que Siempre. De igual manera le $18,52 \%$ de las mujeres y de los hombres asumen que Frecuentemente se les da a conocer la temática a trabajar.

Resulta significativo que en su gran mayoría los docentes vinculados a la investigación presentaron unos ejes temáticos claros $\mathrm{y}$ además los confirman en la entrevista realizada, aunque dos instituciones presentaron la programación no en condiciones óptimas, los 8 docentes restantes las presentaron con diseños básicos que las caracterizaron, lo cual es un indicador del rigor académico con que se elaboran.

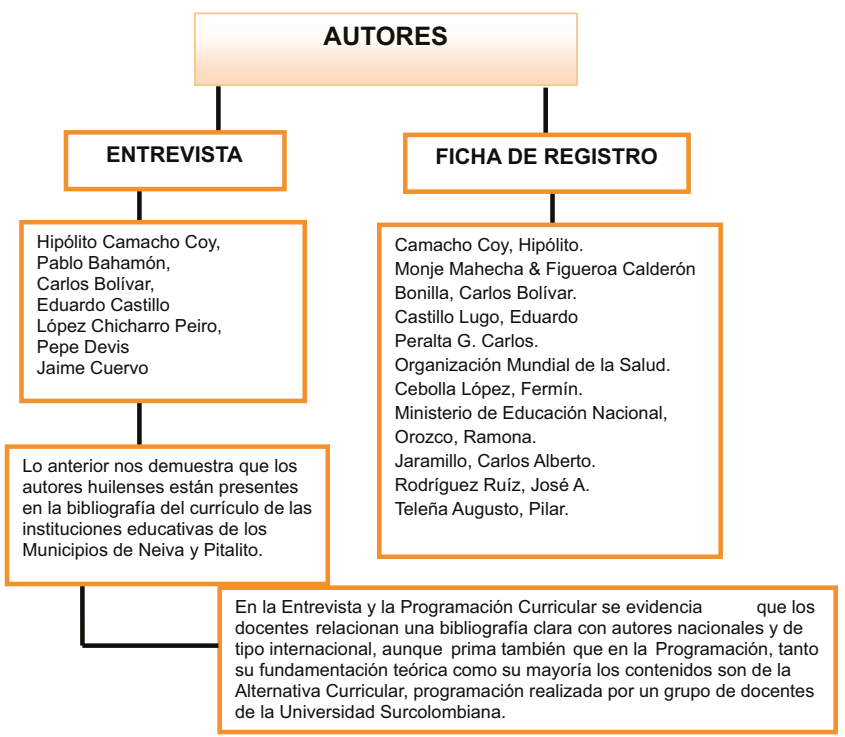

Mapa Conceptual 5. Relación de Autores para realizar la Programación

Aunque en la consulta de autores para la construcción del PEI hay una buena citación bibliográfica, en el momento del desarrollo de las clases de Educación Física los docentes se quedan cortos en el manejo de la conceptualización de las actividades y se falla realizando la misma clase deportivista de siempre.

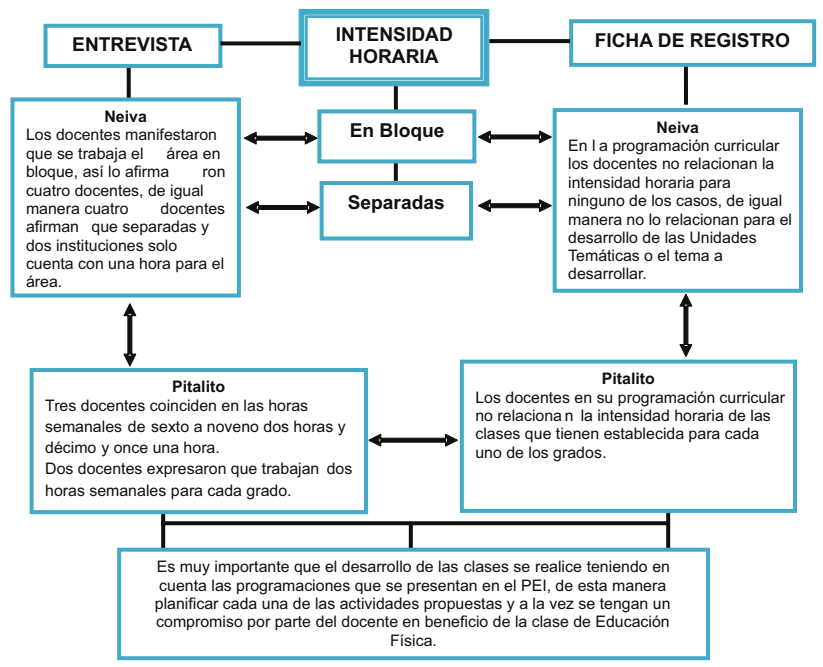

Mapa Conceptual 6. Intensidad Horaria Utilizada para el Área

Además de la periodicidad e intensidad semanal de la clase, es necesario tomar decisiones acertadas sobre la ubicación de ésta en el horario; los criterios para determinar la hora de la clase deben ser de carácter pedagógico, fisiológico y ambiental. 
La tendencia actual en las instituciones educativas es la de programar un bloque de dos clases en una sola sesión semanal cuya duración oscila entre 100 y 120 minutos. Argumentando razones de tipo administrativo en la medida que se facilita la confección de los horarios y el uso del uniforme de Educación Física entre otros aspectos.

En síntesis, en la definición de horarios y periodos de clase de Educación Física, deben primar los criterios científicos, técnicos y pedagógicos sobre los administrativos. Aquí es necesario que el profesor del área tenga argumentos sólidos para orientar de la manera más adecuada la toma de decisiones.

\section{Métodos y Formas de Trabajo}

Los Métodos y Formas de Trabajo vienen de la mano con todo lo relacionado a las áreas del conocimiento y específicamente al área de Educación Física; se refiere al trabajo del profesor de Educación Física, su forma de desarrollar los contenidos y darle el mejor manejo posible al desarrollo de sus actividades, es significativo los estilos de enseñanza que éste desarrolle en sus clases diarias.

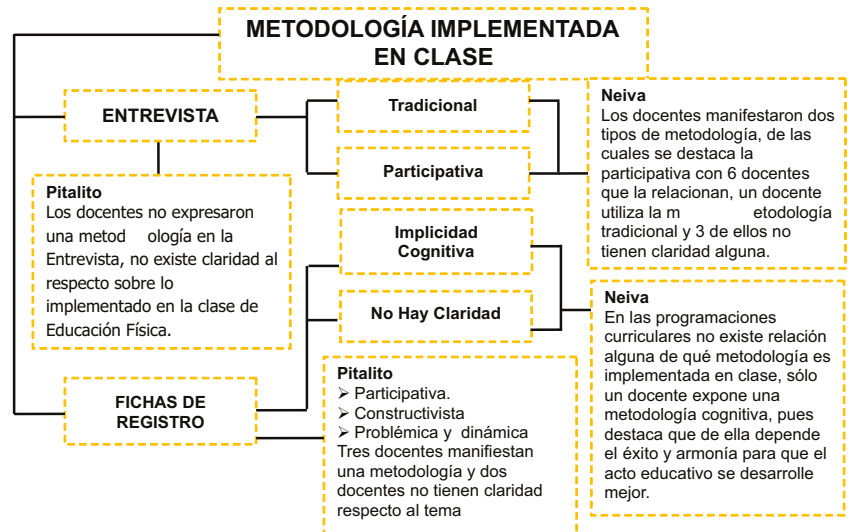

Mapa Conceptual 7. Metodología Implementada en Clase

Luego de hacer el análisis de las Entrevistas y las Programaciones Curriculares, los docentes relacionaron los estilos o una metodología que promueve la participación, sin embargo, en las Programaciones curriculares no se encuentra en el documento una exposición clara sobre la metodología.

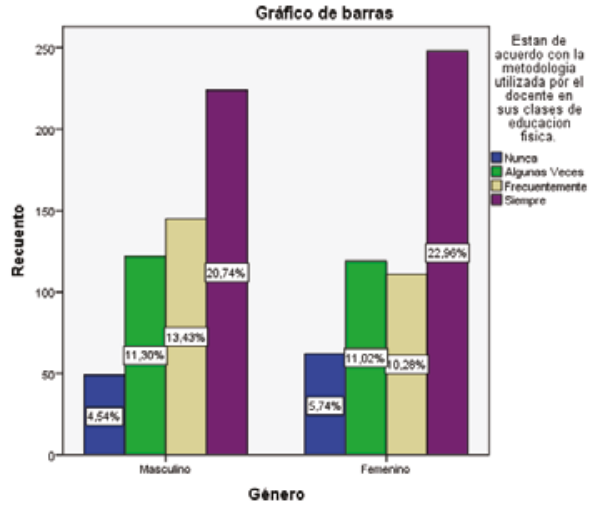

Ilustración 4. Metodología Utiliza por el Docente en el Municipio de Neiva

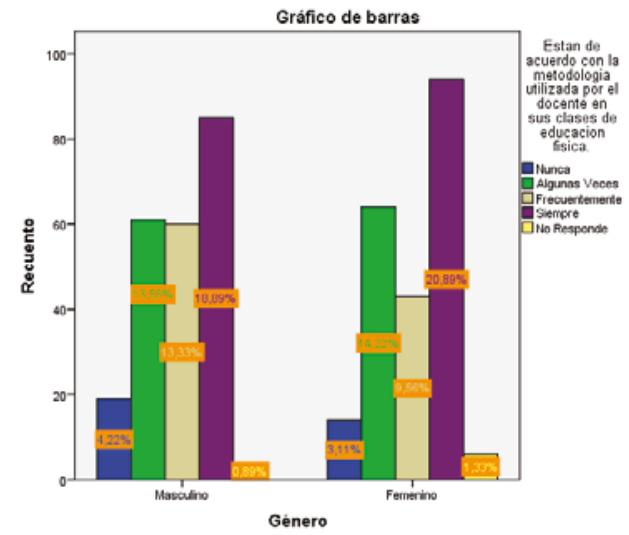

Ilustración 5. Metodología Utilizada por el Docente en el Municipio de Pitalito.

De acuerdo a los datos arrojados en las encuestas, para el Municipio de Neiva los estudiantes se sienten a gusto con la metodología que el docente implementa en clase, aunque éste no tiene claridad en la programación de cuál utiliza específicamente. Para ellos es importante y sienten gozo con sus clases de Educación Física, así lo demuestra el 43,7\% entre hombres $\mathrm{y}$ mujeres que se sienten a gusto y satisfechos con forma en que las clases son impartidas. El $23.71 \%$ de los estudiantes (hombres y mujeres) afirman que Frecuentemente se sienten a gusto, y el porcentaje restante (31.56\%) para Algunas Veces y Nunca.

Para el Municipio de Pitalito, se encontró que el $39,78 \%$ de las mujeres y los hombres manifestaron que Siempre. Con la opción Frecuentemente los hombres y mujeres dan el $22,89 \%$ con un resultado elevado Algunas Veces muestra que las mujeres lo expresaron con un 
$14,22 \%$ y los hombres 13,56. La opción Nunca es un poco más alta en hombres con un $4,22 \%$ y en las mujeres más bajo con 3,11\%.

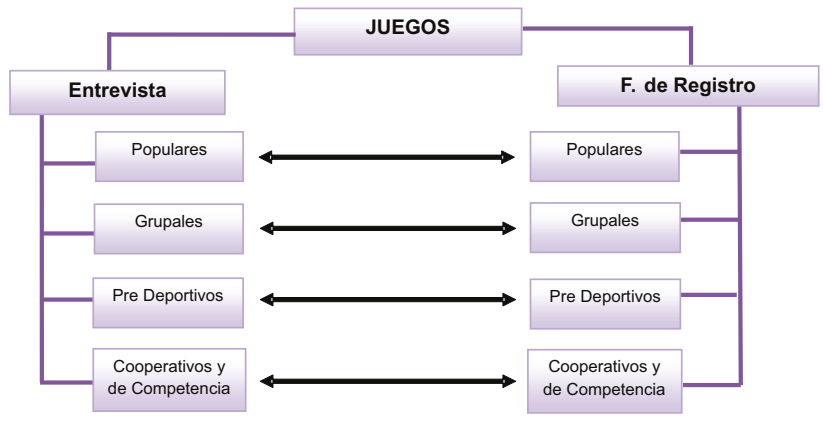

Mapa Conceptual 8. Qué tipo de Juegos Implementa en la Clase

Cuando se habla de juegos o actividades que desarrolla el docente en sus clases de Educación Física, no existe coherencia alguna en lo que manifestaron los docentes en las entrevistas y lo plasmado en las Programaciones Curriculares; tanto para los Municipios de Neiva y Pitalito, hacen uso de juegos Populares, Grupales, Predeportivos, Cooperativos y de Competencia, aspecto que se hace necesario para que las clases sean motivadoras, de gozo, de participación activa, de armonía y de integración grupal por parte de cada estudiante.

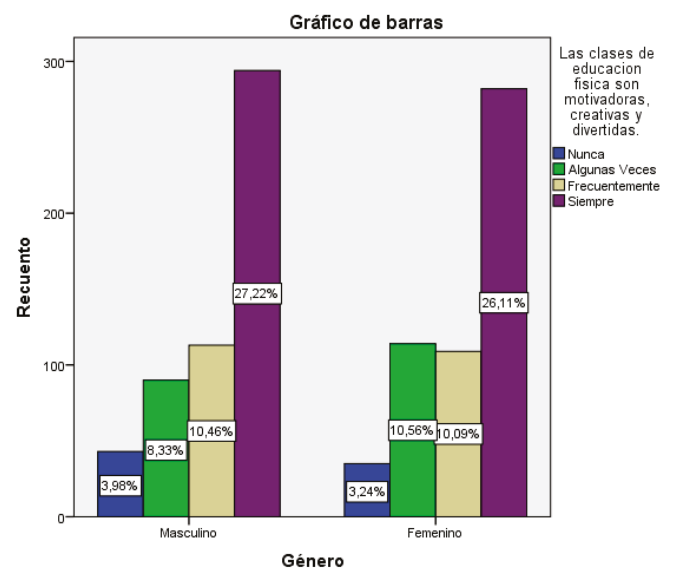

Ilustración 6. Las clases son Motivadoras Creativas y Divertidas en el Municipio de Neiva

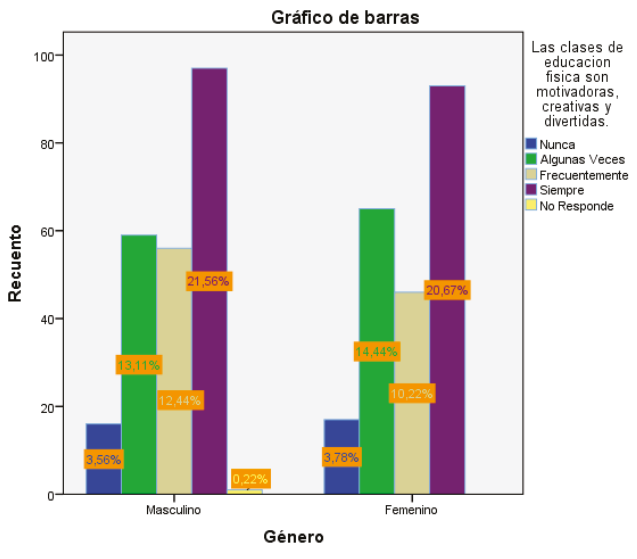

Ilustración 7. Las clases son Motivadoras Creativas y Divertidas en el Municipio de Pitalito

Luego de los datos arrojados en las encuestas, los estudiantes del Municipio de Neiva manifiestan que Siempre las clases del docente de Educación Física son motivadoras, creativas y divertidas, así lo afirman estudiantes hombres y mujeres con un 53,33\% (más del $50 \%$ de la población en total); igualmente estudiantes hombres y mujeres con un $20,55 \%$ responden Frecuentemente. Es decir que Nunca y Algunas Veces solo suman un 26,12\%. Los resultados arrojados para el Municipio de Pitalito, en la pregunta: las clases de Educación Física son motivadoras, creativas y divertidas, se encontró que el $42,23 \%$ en las mujeres y los hombres dicen que Siempre; en la opción Frecuentemente muestra un $22,66 \%$ en género femenino $y$ masculino; Algunas Veces arrojó un porcentaje del $27,55 \%$ en femenino y en masculino.

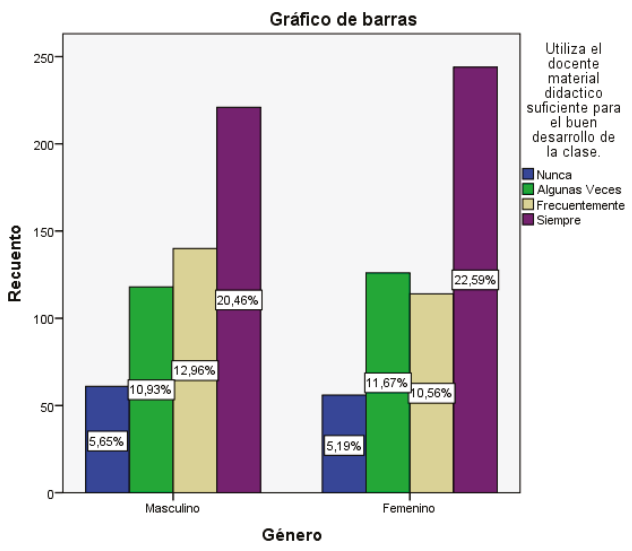

Ilustración 8. Material Deportivo Municipio Neiva 


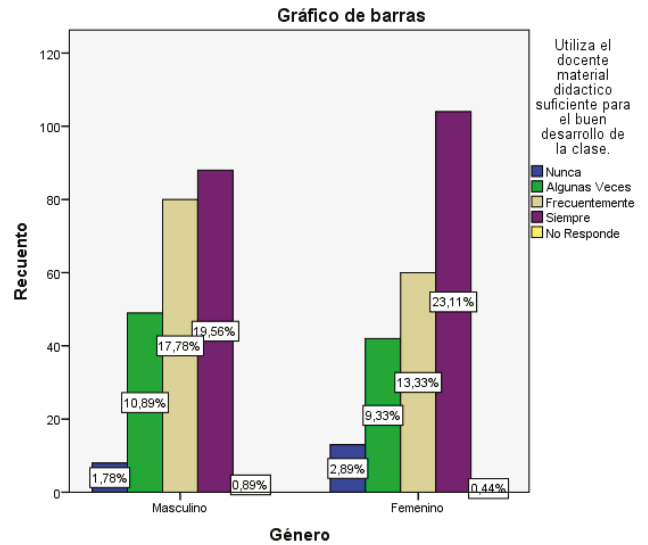

Ilustración 9. Material Deportivo Municipio Pitalito

Los datos arrojados en las encuestas demuestran que los estudiantes manifiestan que Siempre el docente utiliza material didáctico necesario para el desarrollo de sus clases -así lo afirma el $43,05 \%$ de los y las estudiantes-, con un 23,52\% para Frecuentemente, de igual manera entre hombres y mujeres; es decir que Nunca y Algunas Veces solo suman un 33.43\% que obedece a instituciones con un escenario deportivo no adecuado y con la falta de implementación deportiva. Para el Municipio de Pitalito, las mujeres con el 23,11\% y en hombres el $19,56 \%$, manifiestan que Siempre; para la opción Frecuentemente los hombres respondieron en un $17,78 \%$ y las mujeres el 13,33\%; Algunas Veces muestra una pequeña diferencia, pues mientras que en las mujeres se da el $9,33 \%$ en los hombres esta el 10,89\%, y finalmente la opción Nunca nos arrojó el 2,89\% para género femenino y $1,78 \%$ para el género masculino.

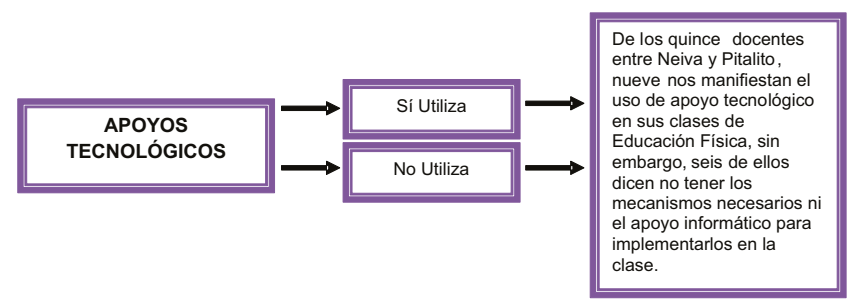

Mapa Conceptual 9. Apoyos Tecnológicos para la Clase Municipios de Neiva y Pitalito

Con la recopilación de información, se puede afirmar que las instituciones educativas utilizan apoyos tecnológicos en su clase, tales como video beam, reproducción de video, acceso a las páginas de internet y portales educativos en Educación Fisca. Por el contrario en la actualidad son pocos los docentes que no utilizan apoyos tecnológicos porque manifiestan no haber nada para el área, y que solo es necesario para las demás asignaturas.

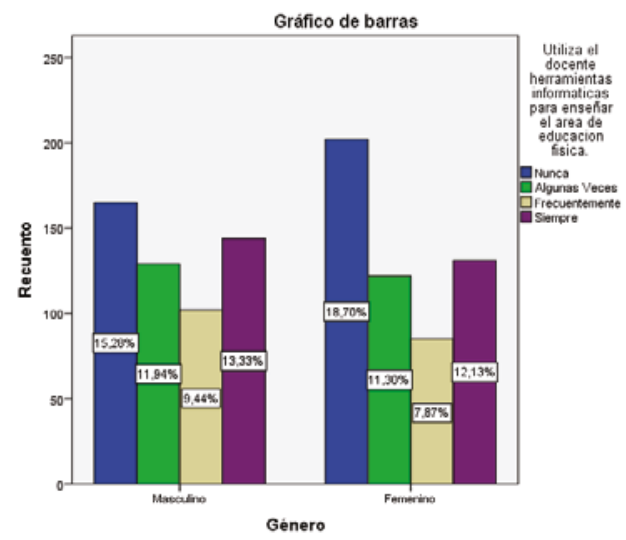

Ilustración 10. Herramientas Municipio de Neiva

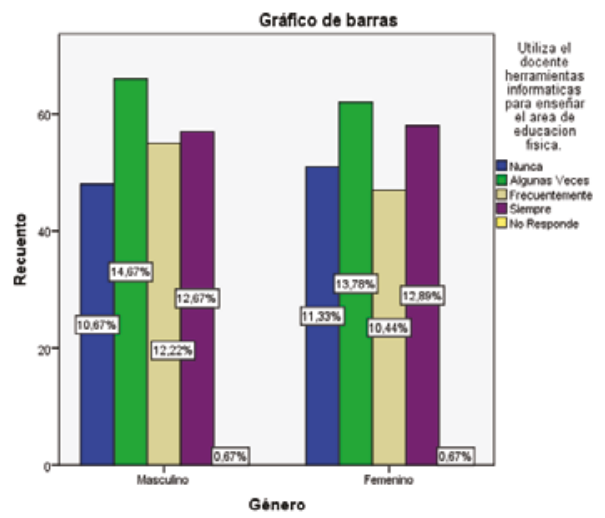

Ilustración 11. Herramientas Municipio de Pitalito

Es evidente la falta de uso de apoyo tecnológico en el área para el Municipio de Neiva: El 25,46\% de las mujeres y de los hombres dicen que Siempre las implementa; para Frecuentemente responden el $17,31 \%$ de las mujeres y de los hombres; el 23,24\% de mujeres y de hombres dicen que Algunas Veces; y Nunca ocupa el porcentaje más alto tanto en hombres como en mujeres con un 33,98\% que corroboran que el docente solo les orienta el área en el campo deportivo y/o el salón de clase pero sin estas ayudastecnológicas. Parael Municipio dePitalito, en la gráfica se evidencia que con un $25,56 \%$ en el género femenino y masculino, expresaron que Siempre; Frecuentemente arrojo un $22,66 \%$ para 
mujeres y hombres; Algunas Veces fue la opción más utilizada por los encuestados, las mujeres y los hombres con un 28,35\%; la opción Nunca tuvo también un porcentaje alto el $22 \%$ para los hombres y mujeres.

\section{Evaluación}

La evaluación es uno de los aspectos más significativos en el área de Educación Física, dado que es allí donde el docente interactúa directamente con el estudiante de una manera práctica y teórica, es por ello que se da a conocer acerca de la conceptualización y los criterios de evaluación que el docente utiliza, así como también lo que los estudiantes de las instituciones perciben del maestro en los procesos evaluativos para el área.

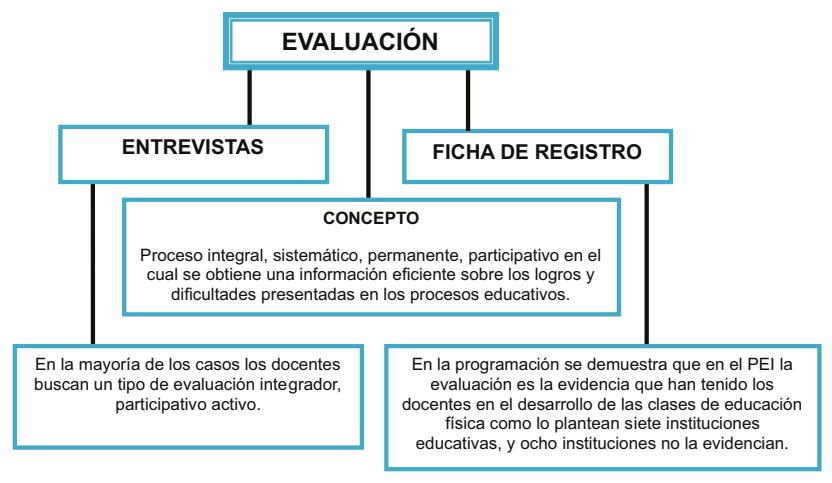

Mapa Conceptual 10. Conceptualización de Evaluación

En la variable que hace alusión a la Evaluación, se aprecia que en las instituciones educativas de los municipios de Neiva y Pitalito, se implementa la evaluación como requisito dado por los entes educativos; sin embargo, se evidencia que falta una mayor fundamentación en este tema por parte de los docentes. Aunque se está de acuerdo en la posibilidad de que los establecimientos tuvieran un mismo modelo y no se dificultara los procesos educativos sino más bien que existiera un mismo criterio para todas las Instituciones, también se sugiere la necesidad de que el modelo de evaluación, aunque sea similar debe tener en cuenta los aspectos relacionados con el contexto. Pero lo más importante es que el Educador Físico tenga claridad con dicho modelo y que sea pertinente a su propia institución.

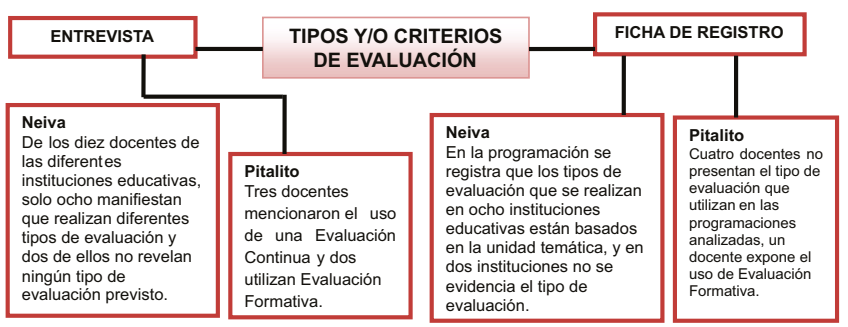

Mapa Conceptual 11. Tipos y Criterios de Evaluación

En las instituciones educativas del Municipio de Neiva existe coherencia en las entrevistas realizadas y en las programaciones curriculares analizadas, dado que en ambos casos se encontró alguna relación con los tipos de evaluación implementados en el área; varios docentes tienen en cuenta aspectos como: puntualidad, comportamiento, aseo, disciplina, responsabilidad, valores fundamentales para el buen desarrollo del área y que le permiten crear los criterios de evaluación y así adecuarlos al contexto, además es notable que su evaluación es práctica, participativa y como eje fundamental la formación integral de los estudiantes.

Para el Municipio de Pitalito, cada docente según su formación y criterio utiliza diversos tipos de evaluación, como lo son la Formativa y la evaluación Continua. Claramente se evidencia que en las instituciones educativas del Municipio de Pitalito, falta capacitar a los docentes encargados de orientar el área en el aspecto que hace alusión a la evaluación, para que el proceso de enseñanza aprendizaje mejore. Los docentes bajo sus criterios definen cual es el tipo de evaluación que pueda medir el aprendizaje de sus estudiantes. Este debe ser flexible a las situaciones con la intención de hacer de ella un proceso de formación en los educandos.

A continuación, se darán a conocer algunas percepciones que tienen los estudiantes acerca de la Evaluación que implementa el docente en el área, de cómo los evalúa, esto con el fin de conocer sus apreciaciones y en qué concepto valoran tanto al docente como a la asignatura. 


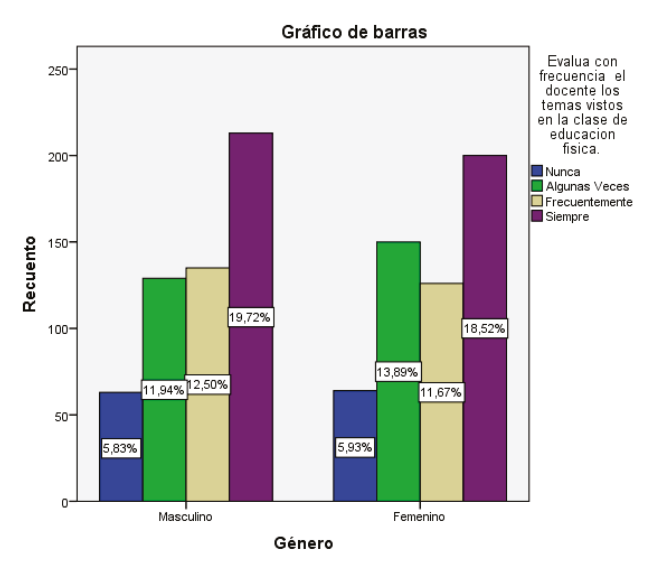

Ilustración 12. Evalúa con frecuencia el docente los temas en la clase de Educación Física en el Municipio Neiva

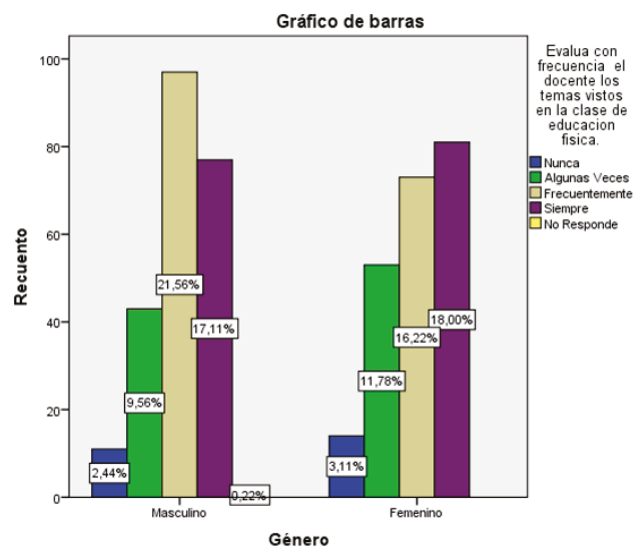

Ilustración 13. Evalúa con frecuencia el docente los temas en la clase de Educación Física en el Municipio Pitalito

$\mathrm{Al}$ analizar este aspecto, en un gran porcentaje el docente evalúa con frecuencia los temas vistos en clase, así los afirman en su gran mayoría los estudiantes de las Instituciones Educativas Públicas de Neiva, con un 38,24\% mujeres y hombres que dicen Siempre; Frecuentemente lo dicen el 24,17\% de las mujeres y de los hombres; para Algunas Veces, las mujeres y hombres con el $25.83 \%$; y Nunca solo tiene un porcentaje del 11,76\% para Mujeres y para Hombres. Es necesario saber qué tan bueno es o no evaluar frecuentemente los temas vistos por parte del docente de educación física. Para el Municipio de Pitalito, la opción Siempre las mujeres y los hombres mostraron un 35,11\%; Frecuentemente arrojó el 37,78\% para hombres y mujeres; para Algunas Veces un 21,34\% en mujeres y hombres, el 5,55\% de los hombres y las mujeres fue el resultado para la opción Nunca.

Lo anterior nos confirma que, aunque el área de Educación Física es práctica, el docente evalúa cualquier aspecto que orienta y esto lo hace continuamente.

\section{Conclusiones}

La adopción de una determinada tendencia ésta condicionada por factores como los objetivos y los valores a formar en el estudiante, los que a su vez están soportados por una finalidad preconcebida a nivel político y filosófico, ante esta situación el predominio de una tendencia responde a factores contextuales que la delimitan, y también la explica.

Ante estos preceptos, la tendencia utilizada por un docente, debe ser argumentada a nivel teórico, para conocer qué, criterios llevan a su implementación, ya que de esta manera se aprecia una clara intención y no una inercia entendida como una rutina sin ninguna dinámica, producto de hacer lo mismo sin ningún juicio valorativo ni un criterio autocrítico que desdice del ejercicio docente.

Desafortunadamente, la investigación encontró una marcada tendencia deportivista, sin otra explicación que una marcada preferencia de los muchachos, sin intentar darle un valor agregado, un enfoque diferente que provea al estudiante de la inmensa riqueza que puede aportar la Educación Física al desarrollo humano. Ninguna tendencia es buena o mala, cada una de éstas presenta sus ventajas y desventajas, lo ideal es que el docente encargado de incorporarla en sus clases, cuente con una fundamentación teórica que la defienda en la práctica y la teoría.

El desarraigo de una tendencia es muy difícil, sobre todo algunas que llevan siglos siendo implementadas, reforzadas con programas gubernamentales que las alientan como la deportivista, por eso muchas instituciones ven la competencia deportiva como representación, y el docente es valorado por la medalla que gana en los diferentes juegos que organizan las instituciones deportivas o directamente 
Coldeportes Departamental y no por la atención a todos los estudiantes en la clase. Lo triste es que no solo es deportivista, sino que hay un predominio por el fútbol y el fútbol de salón, con muy poca diversificación.

Así lo confirman también los estudiantes, pues según los datos arrojados en la Encuesta se estableció que en su gran mayoría tanto mujeres como hombres, afirman que la tendencia utilizada es la Deportivista, diciendo que Siempre el docente desarrolla actividades deportivas y/o fundamentación técnica en el área de Educación Física.

Se observan inclinaciones también hacia una tendencia expresiva, que puede enriquecer la clase de Educación Física, pero esto solo es posible si la Universidad Surcolombiana de donde provienen la mayoría de docentes hace sus propias innovaciones dentro del plan de estudios, inculcando una mentalidad más abierta a sus estudiantes, haciendo una continua actualización a sus egresados a través de un programa de proyección social que mantenga viva la motivación por el área. Además, los postgrados tan necesarios a nivel académico como administrativo para los egresados, tienen como imperativo suministrar elementos novedosos, incluyendo renovados conceptos teóricos.

Es posible que haya tendencias apropiadas para determinados grupos etarios, por ejemplo, una concepción psicomotriz tiene más cabida en la escolaridad primaria que en la secundaria, pero la tendencia expresiva puede tener éxito en más grados de escolaridad. La intencionalidad de la utilización de determinada tendencia pasa también por los recursos que se piden a la institución, si solo se piden balones, eso es lo que el rector suministra. Tenemos una ardua tarea en el intento de diversificar y utilizar el potencial formador de la educación física en toda su dimensión para las generaciones venideras.

En las programaciones citadas no se evidencian de manera explícita criterios en los contenidos, metodología y evaluación claras y precisas, de esta manera, en su mayoría los docentes del área de Educación Física nos expresaron una asignatura completa y al corroborar esto en las Programaciones Curriculares no se encontró conexión con los Aspectos fundamentales del área.

No obstante, luego del análisis, se observa que existe coherencia por parte de los docentes tanto en las programaciones curriculares como en la entrevista, dado que trabajan los mismos ejes temáticos para el área, ejes temáticos planteados en la Alternativa Curricular del área de Educación Física, por docentes de Educación Física de la Universidad Surcolombiana. Se concluye que existe directa relación entre lo que se desarrolla en el campo deportivo y lo planteado en las programaciones curriculares.

Uno de los aspectos más reveladores de la acción docente y de la importancia que le otorga a la Educación Física que orienta, es la evaluación, un área que tiene muchas variables a tener en cuenta, se ve reducida a una especie de entrenamiento deportivo, y en esa medida los tiempos empleados $\mathrm{o}$ las repeticiones realizadas, todavía son criterios de objetividad en la evaluación, quiere decir que el propósito formativo no es tenido en cuenta, y tras una pretendida neutralidad técnica, se traslada un sentimiento de culpa al estudiante menos hábil que es el que más necesita de una verdadera educación física.

Este tipo de evaluación, termina alejando al estudiante de la Educación Física, hay aspectos trascendentales que no se valoran como las características personales, afectivas, sociales y contextuales del estudiante. Evaluar estos aspectos exige al docente una especial sensibilidad, para observar y valorar lo cualitativo, y hace parte de una responsabilidad social del área con el futuro ciudadano, esto explica en parte por qué no son suficientes estos seis años de escolaridad para formar hábitos saludables como la práctica de la actividad física una vez se concluya la media vocacional.

Entre educar el movimiento y entrenar cuerpos hay una buena diferencia, la Educación Física puede estimular la creación de liderazgo no necesariamente con base en las facilidades 
atléticas de un estudiante, además, hay otros escenarios en los cuales no ha sido estimulado el estudiante, un estudiante pasivo en la clase de educación física puede ser un gran parejo de Sanjuanero, o un destacado malabarista, pero si solo se utiliza una predeterminada programación, si no se contemplan otras opciones, se pueden cercenar posibilidades a los estudiantes.

Con respecto al estudiante: éste reconoce que el área de Educación Física es valorada y apreciada por ellos, así lo manifestaron las encuestas aplicadas. Además de querer indagar por el área, se preguntó por el docente, acerca de cómo la orienta y de cómo se sienten ellos al recibir la asignatura, obteniendo resultados significativos en los que el docente juega un papel importante en la Institución, es apreciado por el estudiante y nos comunican el querer solicitar un mayor número de horas para el área.

\section{Referencias bibliográficas}

Camacho Coy, H., \& Amaya Bahamon, L. (2011). Una mirada al Área de Educación Física en el Departamento del Huila. Entornos, 123137.

Camacho Coy, H. (2003). Pedagogía y Didáctica de la Educación Física (Primera ed., Vol. 1). Armenia, Quindio: Kinesis.

Camacho Coy, H., Castillo Lugo, E., Monje Mahecha, J., \& Ramirez López, G. M. (2008). Competencias Y Estándares para la Educación Física. Una Experiencia que se Construye paso a paso. Armenia: Kinesis.

Camacho Coy, H., Bonilla Baquero, C. B., Quiñonez Toledo, J. D., Castillo Lugo, E., \& Yucuna Vela, Y. (2004). El Imaginario del Adolescente ante la Clase de Educación Física. Neiva: Univerisdad Surcolombiana .

Camacho Coy, H., Castillo Lugo, E., \& Monje Mahecha, J. (2007). Educación Física Programas de $6^{\circ}$ a $11^{\circ}$, Una Alternativa Curricular. Neiva: Kinesis.
Camacho Coy, H., Guzmán Rodríguez , J. D., \& Amaya Bahamón, L. P. (2012). Estrategías Pedagógicas para el Desarrollo de Competencias Ciudadanas desde la Clase de Educación Física. Neiva: Kinesis.

Cantonini Rebolini, N. (2009). Técnicas de Muestreo y Determinación del Tamaño de la Muestra en Investigación Cuantitativa. Revista Argentina de Humanidades y Ciencias Sociales, 10.

Cipriano, R. C., \& Salicetti, F. A. (2009). La contribución del trabajo grupal de los estudiantes como estrategia docente en la formacion del maestro especialista en educacion fisica. Revista Iberoamericana de educación, 11-12.

Devis Devis , J., \& Piero Velert, C. (1992). Nuevas Perspectivas Curriculares en Educación Física: La Salud y Los Juegos. España: Inde Publicaciones.

García Carrillo, L. S. (2015). La Evaluación Formativa ¿Un Concepto en Algunos Casos, Difuso e Impreciso o Una Práctica en el Aula. México: Tecnologico de Monterrey.

Granero Gallegos, A., Baena Extremera, A., \& Martínez Molina, M. (2010). Contenidos Desarrollados mediante las Actividades en el Medio Natural de las Clases de Educación Física en Secundaria Obligatoria. Ágora para la Educación Física y el Deporte, 273-288.

Hernández Sampieri, R., Fernández Collado , C., \& Baptista Lucio, M. (2010). Metodología de la Investigación Quinta Edición. México: McGRA Q-HILL /INTERAMERICANA EDITORES, S.A. DE C.V.

Hernandez Sampieri, R., Collado Fernandez, c., \& Baptista Lucio, P. (2006). Metodología de la Investigación. México D.F.: Mc. Graw Hill.

Ministerio de Educación Nacional. (18 de Agosto de 2010). www.ministeriodeeducacion.gov. co. Obtenido de www.ministeriodeeducacion. 
ENTORNOS, Vol. 30, No. 2, Noviembre 2017

gov.co/serielineamientoscurriculares/ preescolar

Ministerio de Educación Nacional. (2000). Educación Física Recreación y Deportes. Serie. Lineamientos Curriculares. Bogotá D. C: Magisterio.

Morales Rincón, C. A. (2014). Una Mirada a la Educación Física en Colombia. EF Deportes.com, 1-18.

Moreno Olivos, T. (2011). La Cultura de la Evaluación y la Mejora de la Escuela. Perfiles Educativos, vol XXXIII núm 131, 116-130.

Nerici citado en Mena 1997 citado por Hernández, N. B. (2009). Los métodos de enseñanza en la Educacion Fisica. Los métodos de enseñanza en la Educación Física, 1.

Pérez Iribar, G. (2012). Estudio sobre las Principales Tendencias Pedagógicas Contemporáneas en el Proceso de Enseñanza Aprendizaje de la Educación Física. Buenos Aires: EF Deportes.

Pública, Secretaria de Educación. (2004). Programas de Educación Física Preescolar, Primaria y Secundaria. México: Unidad de Informática de la DGEF.

Rodriguez, G. (1996). Metodología de la Investigación Cualitativa. Bogotá: Paidos. Secretaría de Educación Pública. México D.F. (2004). Programas de Educación Física Preescolar, Primaria y Secundaria. México D.F: Unidad de informática de la DGEF. 